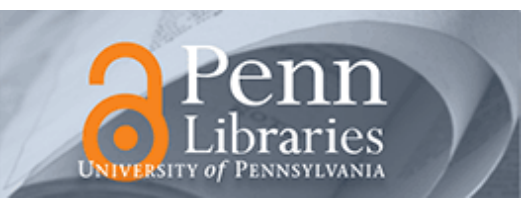

University of Pennsylvania

ScholarlyCommons

$9-1-2009$

\title{
Power Handling and Related Frequency Scaling Advantages in Piezoelectric AIN Contour-Mode MEMS Resonators
}

Chengjie Zuo

University of Pennsylvania, chengjiezuo@hotmail.com

Matteo Rinaldi

University of Pennsylvania, rinaldim@seas.upenn.edu

Gianluca Piazza

University of Pennsylvania, piazza@seas.upenn.edu

Follow this and additional works at: https://repository.upenn.edu/ese_papers

Part of the Electrical and Electronics Commons

\section{Recommended Citation}

Chengjie Zuo, Matteo Rinaldi, and Gianluca Piazza, "Power Handling and Related Frequency Scaling Advantages in Piezoelectric AIN Contour-Mode MEMS Resonators", 2009 IEEE International Ultrasonics Symposium (IUS 2009), 1187-1190. September 2009.

Suggested Citation:

Chengjie Zuo, Matteo Rinaldi, and Gianluca Piazza. "Power Handling and Related Frequency Scaling Advantages in Piezoelectric AIN Contour-Mode MEMS Resonators" 2009 IEEE International Ultrasonics Symposium (IUS 2009) (2009): 1187-1190.

(C2009 IEEE. Personal use of this material is permitted. However, permission to reprint/republish this material for advertising or promotional purposes or for creating new collective works for resale or redistribution to servers or lists, or to reuse any copyrighted component of this work in other works must be obtained from the IEEE.

This paper is posted at ScholarlyCommons. https://repository.upenn.edu/ese_papers/542

For more information, please contact repository@pobox.upenn.edu. 


\title{
Power Handling and Related Frequency Scaling Advantages in Piezoelectric AIN Contour-Mode MEMS Resonators
}

\author{
Abstract \\ This paper reports on the analytical modeling and experimental verification of the mechanically-limited \\ power handling and nonlinearity in piezoelectric aluminum nitride (AIN) contour-mode resonators (CMR) \\ having different electrode configurations (thickness field excitation, lateral field excitation, one-port and \\ two-port configurations) and operating at different frequencies (177-3047 MHz). Despite its simplicity, the \\ one-dimensional analytical model fits the experimental behavior of AIN CMRs in terms of power handling \\ capabilities. The model and experiment also confirm the advantage of scaling (i.e. miniaturizing) the AIN \\ CMRs to higher frequencies at which higher critical power density can be more easily attained up to \\ values in excess of $10 \mu \mathrm{W} / \mu \mathrm{m} 3$.

\section{Disciplines} \\ Electrical and Electronics

\section{Comments} \\ Suggested Citation: \\ Chengjie Zuo, Matteo Rinaldi, and Gianluca Piazza. "Power Handling and Related Frequency Scaling \\ Advantages in Piezoelectric AIN Contour-Mode MEMS Resonators" 2009 IEEE International Ultrasonics \\ Symposium (IUS 2009) (2009): 1187-1190. \\ (C2009 IEEE. Personal use of this material is permitted. However, permission to reprint/republish this \\ material for advertising or promotional purposes or for creating new collective works for resale or \\ redistribution to servers or lists, or to reuse any copyrighted component of this work in other works must \\ be obtained from the IEEE.
}




\title{
Power Handling and Related Frequency Scaling Advantages in Piezoelectric AlN Contour-Mode MEMS Resonators
}

\author{
Chengjie Zuo, Matteo Rinaldi, and Gianluca Piazza \\ Department of Electrical and Systems Engineering \\ University of Pennsylvania \\ Philadelphia, PA 19104, USA \\ \{czuo, rinaldim, piazza\}@ seas.upenn.edu
}

\begin{abstract}
This paper reports on the analytical modeling and experimental verification of the mechanically-limited power handling and nonlinearity in piezoelectric aluminum nitride (AIN) contour-mode resonators (CMR) having different electrode configurations (thickness field excitation, lateral field excitation, one-port and two-port configurations) and operating at different frequencies (177-3047 MHz). Despite its simplicity, the onedimensional analytical model fits the experimental behavior of AIN CMRs in terms of power handling capabilities. The model and experiment also confirm the advantage of scaling (i.e. miniaturizing) the AIN CMRs to higher frequencies at which higher critical power density can be more easily attained up to values in excess of $10 \mu \mathrm{W} / \mu^{3}$.
\end{abstract}

Keywords - power handling; nonlinearity; frequency scaling; AlN contour-mode resonator; microelectromechanical systems (MEMS)

\section{INTRODUCTION}

Defining power handling limits of a resonator is of paramount importance since it sets the type of application in which a filter can be used and directly affects the phase noise of oscillators. As an interesting topic under significant study for decades, the power handling capability of a resonator has been found to be correlated to different phenomena in different technologies. For example, anelastic material properties dominate for quartz crystal resonators [1], electrode acoustic migrations for surface acoustic wave (SAW) devices [2], and catastrophic failure for thin film bulk wave acoustic resonators (FBAR) [3].

On the other hand, thanks to the great potential for integration with CMOS integrated circuits and multi-frequency operation up to several $\mathrm{GHz}$ on a single chip, Micro/Nano ElectroMechanical Systems (MEMS/NEMS) resonators have emerged as a very promising alternative technology to replace conventional quartz crystal and SAW devices in future wireless communication and sensing applications. Based on either electrostatic $[4,5]$ or piezoelectric $[6,7]$ transduction, MEMS resonators have been recently demonstrated to simultaneously attain high quality factor $(Q>1,000)$ and high operating frequencies up to $\mathrm{GHz}$. The power handling and nonlinearity of Si-based electrostatic MEMS resonators has been previously studied [8-10] and it shows that the power handling capability of Si-MEMS resonators is generally lower than conventional quartz crystal devices because of the smaller size of the device (therefore lower energy storage capacity), nonlinear effects in the material properties and especially the use of electrostatic transduction.

Compared with electrostatic (capacitive) transduction for MEMS, piezoelectric transduction is considered to be much more linear. It has also been shown that the piezoelectric coefficient $\left(d_{31}\right.$ and $\left.d_{33}\right)$ of aluminum nitride (AlN) keeps linear even under large electric fields [11]. For this reason, mainly material nonlinearity needs to be considered for piezoelectric MEMS resonators. In the piezoelectric AlN contour-mode resonators (CMRs) [6, 7, 14], the presence of thin electrodes should also be taken into account, since it can ultimately limit power handling capability as in SAW devices. This aspect is not studied in this paper since it was not experimentally encountered. It is also expected that electro migration will be less of a problem in CMRs than in SAW devices since CMRs can resort to different electrode materials and generally use wider electrodes than in SAW transducers. Although it is well recognized that piezoelectric transduction offers both lower impedances and better power handling than electrostatic devices [12], little work has been done to tackle the problem analytically, and very few experimental verification have been reported [13]. In this paper we report on the systematic analysis of nonlinearity and power handling in piezoelectric AIN CMRs for different electrode configurations and a wide range of operating frequencies. A simple one-dimensional analytical expression that describes power handling in AlN CMRs is derived, verified experimentally and used to find the fundamental limiting physics behind it.

\section{NONLINEAR MODEL}

A simple one dimensional and isotropic analysis is adopted to model the nonlinear mechanical behavior of piezoelectric AlN contour-mode MEMS/NEMS resonators [14]. The model is based on the nonlinear theory of $2^{\text {nd }}$ order mechanical oscillators $[8,15]$. The assumption of describing the resonator behavior with a 1D equation and as an isotropic material can be considered to hold valid for AlN CMRs, since 1D equations describe well (within few \%) their frequency and impedance, and the AlN films are effectively isotropic in the plane of the wafer (main direction of vibration of the resonator). 
The fundamental equations governing forced mechanical oscillations with damping have been studied extensively in the past $[8,15]$. Basically, with the presence of nonlinearities, the amplitude-frequency $(A-f)$ effect is observed [16], and the actual resonance frequency of vibration $\left(\omega_{r}\right)$ is different from the natural frequency $\left(\omega_{0}\right)$ of the device:

$$
\omega_{r}=\omega_{0}\left(1+\kappa_{x} x^{2}\right)
$$

where $x$ is the equivalent displacement of the resonator and $\kappa_{x}$ is the $A-f$ coefficient given by

$$
\kappa_{x}=\frac{3}{8} k_{2}-\frac{5}{12} k_{1}^{2}
$$

where $k_{1}$ and $k_{2}$ are the first-order and second-order correction coefficients of the equivalent spring constant, respectively.

(a)

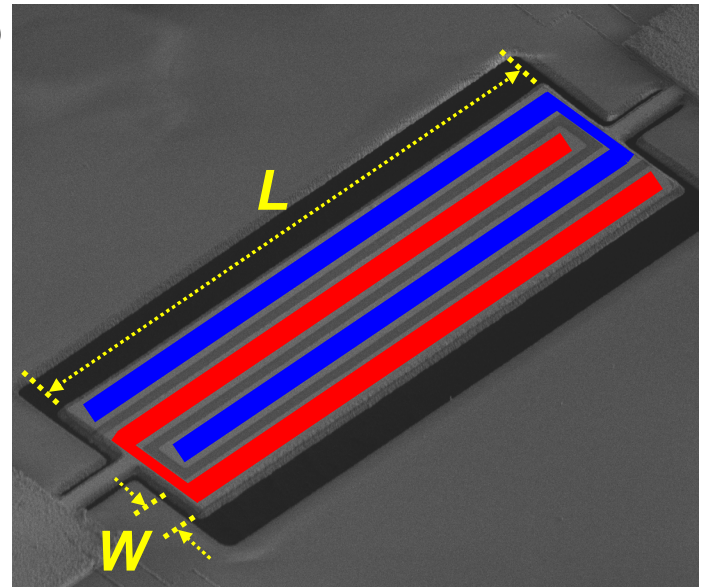

(b)
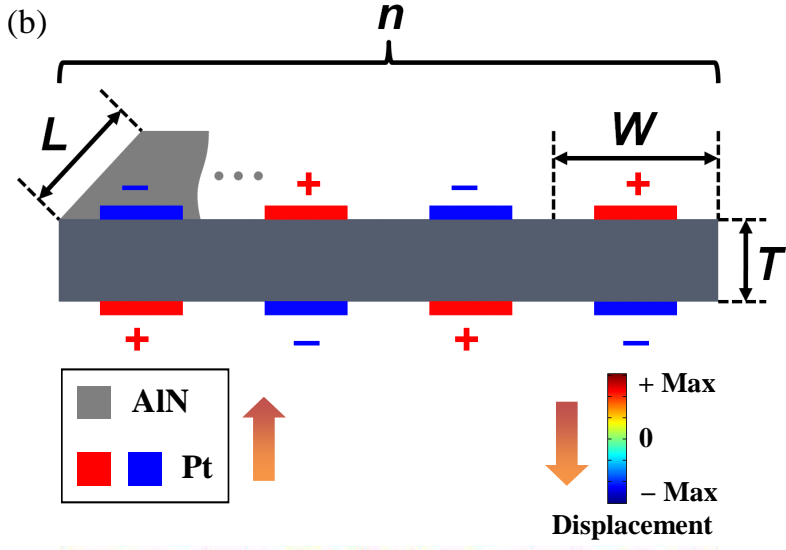

(c)

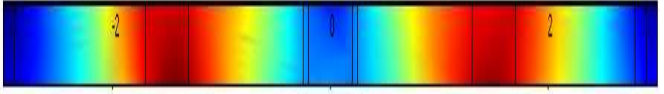

Fig. 1. (a) SEM picture, (b) schematic, and (c) mode shape of a one-port thickness-field-excited (TFE) AlN contour-mode MEMS resonator. $W$ sets the resonator center frequency and corresponds to width of an individual finger. $n$ of these fingers ( 4 in this figure) are coupled to reduce the device impedance.

For a single finger (sub-resonator) in the higher-order oneport thickness-field-excited (TFE) piezoelectric AlN contourmode resonator (with $n$ sub-resonators) $[14,17]$ operating in its fundamental width $(W)$ extensional mode, as illustrated in Fig. 1 , the linear, first-order, and second-order corrections of the spring constant can be expressed as a function of the equivalent nonlinear Young's modulus and the device geometry [8] and consequently the critical vibration amplitude (or the greatest displacement before bifurcation happens) can be expressed as:

$$
x_{c}=\frac{2}{\sqrt{3 \sqrt{3} Q\left|\kappa_{x}\right|}}=\frac{2 \sqrt{2} W}{\sqrt{\sqrt{3} Q\left|9 Y_{2}-10 Y_{1}^{2}\right|}}
$$

where $Q$ is the resonator quality factor and $Y_{0}, Y_{1}$, and $Y_{2}$ are the linear, first-order, and second-order corrections of the strain dependent Young's modulus.

The total root-mean-square (RMS) current flowing through the device (considering $n$ fingers) is related to the resonator equivalent displacement, $x$, by the following equation

$$
i=\frac{1}{\sqrt{2}} n \omega \eta x
$$

where $\omega(=2 \pi f)$ is the angular operating frequency and $\eta$ is the electromechanical coupling factor of a single resonator finger, approximately expressed as

$$
\eta=2 d_{31} Y_{0} L
$$

where $d_{31}$ is the equivalent piezoelectric coefficient of AlN used to transduce vibrations in CMRs. The coefficient is, on the other hand, related to the effective electromechanical coupling coefficient of the material $\left(k_{t}^{2}\right)$ by following approximated expression:

$$
k_{t}^{2}=\frac{d_{31}^{2} Y_{0}}{\varepsilon_{33}}
$$

where $\varepsilon_{33}$ is the permittivity of AlN along the $c$-axis. Although $k_{t}^{2}$ is strictly related to $\eta$, it is a normalized parameter so that it can be conventionally used to describe the figure of merit of a resonator and, for this reason, its use is preferred over $\eta$.

By substituting Eq. (4) into (1), an equivalent currentfrequency coefficient, $\kappa_{i}$, can be derived:

$$
\kappa_{i}=\frac{2}{n^{2} \omega^{2} \eta^{2}} \kappa_{x}=\frac{9 Y_{2}-10 Y_{1}^{2}}{3 n^{2} \omega^{2} \eta^{2} W^{2}}
$$

When the resonator is driven at resonance in the fundamental width extensional mode, the series resonant frequency is [17]

$$
\omega_{s}=2 \pi f_{s}=\frac{\pi}{W} \sqrt{\frac{Y_{0}}{\rho}}
$$

where $\rho$ is the equivalent mass density of $\mathrm{AlN}$ and stacked electrodes (Pt or other materials). By substituting Eq. (8) into (7), an expression for $\kappa_{i}$ that depends exclusively on device geometry and material properties can be derived

$$
\kappa_{i}=\frac{\left(9 Y_{2}-10 Y_{1}^{2}\right) \rho}{12 \pi^{2} Y_{0}^{2} \varepsilon_{33}} \frac{1}{n^{2} L^{2} k_{t}^{2}}
$$


The corresponding critical RMS current is given by

$$
i_{c}=\frac{2}{\sqrt{3 \sqrt{3} Q\left|\kappa_{i}\right|}}=\frac{4 \pi \sqrt{\varepsilon_{33}} Y_{0}}{\sqrt{\sqrt{3} \rho\left|9 Y_{2}-10 Y_{1}^{2}\right|}} \frac{n L k_{t}}{\sqrt{Q}}
$$

Then the critical power (maximum power the resonator can handle before bifurcation occurs) can be derived as

$$
p_{c}=i_{c}^{2} R_{M}=\frac{2 \pi^{3} \sqrt{Y_{0}^{3}}}{\sqrt{3 \rho}\left|9 Y_{2}-10 Y_{1}^{2}\right|} \frac{n L T}{Q^{2}}
$$

where $R_{M}$ is the motional resistance [14, 17]. When a MEMS resonator is driven above the critical power, the bifurcation instability happens [9], which generally induces extra close-tocarrier phase noise in MEMS oscillators or distortion in a filter passband. That is primarily why the critical amplitude is generally taken as a measure of maximum usable vibration amplitude, and the critical power as the maximum power a MEMS resonator can handle [8].

If we divide the critical power by the resonator area, $A$, or volume, $V$, the critical power density per unit area (Eq. (12)) or volume (Eq. (13)) can be found:

$$
\begin{aligned}
& \frac{p_{c}}{A}=\frac{2 \pi^{3} \sqrt{Y_{0}^{3}}}{\sqrt{3 \rho}\left|9 Y_{2}-10 Y_{1}^{2}\right|} \frac{T}{Q^{2}} \frac{1}{W} \propto \frac{T}{Q^{2}} f_{s} \\
& \frac{p_{c}}{V}=\frac{2 \pi^{3} \sqrt{Y_{0}^{3}}}{\sqrt{3 \rho}\left|9 Y_{2}-10 Y_{1}^{2}\right|} \frac{1}{Q^{2}} \frac{1}{W} \propto \frac{1}{Q^{2}} f_{s}
\end{aligned}
$$

Eq. (12) and (13) clearly indicate that the critical power density is inversely proportional to the resonator width. The resonant frequency is also inversely proportional to $W$, as shown in Eq. (8). Assuming the nonlinear coefficients to be a constant, the critical power density (power handling capability per unit area or volume) increases as the TFE AIN contour-mode resonators are scaled to higher frequencies of operation.

\section{DIFFERENT ELECTRODE CONFIGURATIONS}

By properly patterning the bottom and top electrodes in a single-layer AlN CMR, different electrode configurations can be devised for various resonator applications, such as filtering or frequency setting. One and two port TFE devices can be fabricated [17, 18], as well as resonators having interdigitated electrodes only on one surface of the AlN film (lateral field excitation: LFE resonators [19]). The following paragraphs compare the power handling analysis that was derived for a one-port TFE device to the cases for two-port and LFE CMRs.

As a very important element for the synthesis of higherorder self-coupled RF MEMS channel-select filters [18], a twoport TFE AlN resonator has a single electrically grounded $\mathrm{Pt}$ electrode under the entire resonator body, while the top Pt thin film is patterned into interdigitated electrodes, half used for the input port and the other half for the output port, as illustrated in Fig. 2 (a). In this case, the motional current goes into the input port and out of the output port, and the critical current is half the value of a one-port TFE resonator with the same size ( $W, L$, and $T$ ) or volume, since either the input or output port has half the number of sub-resonators. On the other hand, according to the analysis in [18], it is easy to find that the motional resistance of a two-port TFE resonator is four times that of a one-port TFE resonator having the same size. Consequently, we obtain

$$
\begin{gathered}
i_{c, \text { Two-Port, TFE }}=i_{c, \text { One-Port, TFE }} / 2 \\
p_{c, \text { Two-Port, TFE }}=p_{c, \text { One-Port, TFE }}
\end{gathered}
$$

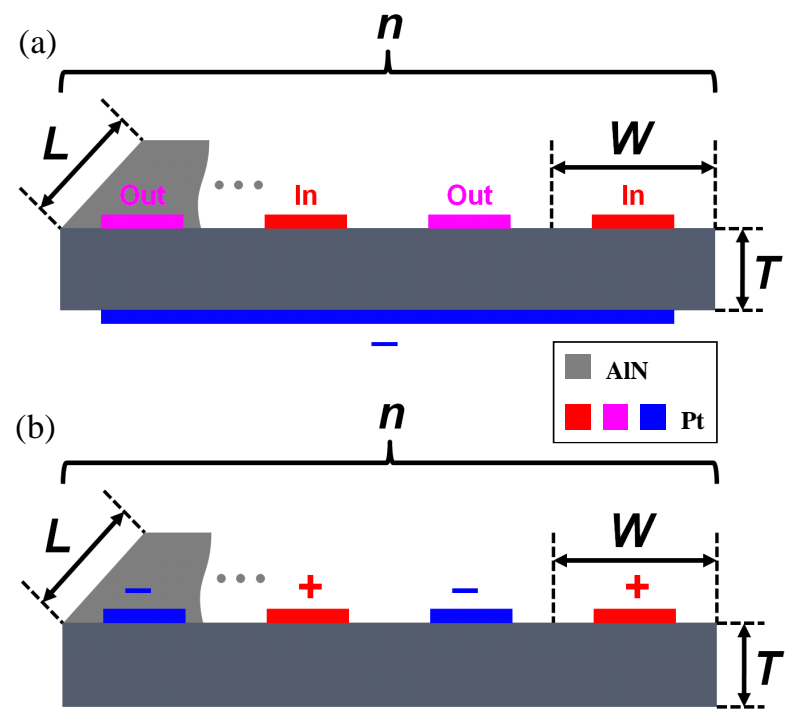

Fig. 2. Cross-sectional schematics of (a) the two-port thickness-field-excited (TFE) and (b) the one-port lateral-field-excited (LFE) AlN contour-mode MEMS resonators.

A similar analysis can be applied to the LFE AlN contourmode resonators [19], for which there are Pt electrodes only on top of the AlN layer. The effect of using LFE (a different transducer) is lumped into the change of $k_{t}^{2}$, while all other parameters stay the same as for TFE resonators. To a $1^{\text {st }}$ order approximation, an LFE resonator has the same AIN structure of a TFE resonator and vibrates in the same mode. It should be noted that for LFE devices there is a dependence of $k_{t}^{2}$ on the ratio, $T / W,[20]$ and the expressions are more complicated. Since the relationship between $k_{t}^{2}$ and $T / W$ is nonlinear, it is not explicitly represented in the following equations, but should be taken into account. Accordingly, the critical current and power for LFE resonators can be expressed as:

$$
\begin{gathered}
i_{c, \text { One-Port, LFE }}=\frac{i_{c, \text { One-Port, TFE }}}{2} \frac{k_{t, \text { One-Port, LFE }}}{k_{t, \text { One-Port, TFE }}} \\
p_{c, \text { One-Port, LFE }}=p_{c, \text { One-Port, TFE }}
\end{gathered}
$$

Therefore, the conclusion is that, to a $1^{\text {st }}$ order approximation, the power handling capability of a piezoelectric AlN CMR is independent of the excitation (or electrode configuration), as long as the same AlN plate is used to make the resonator. However, current handling capability of a CMR does depend on the electromechanical coupling coefficient $k_{t}^{2}$. 
Table I. Experimental Results on Power and Current Handling of Piezoelectric AlN Contour-Mode MEMS Resonators

\begin{tabular}{|c|c|c|c|c|c|c|c|c|c|c|c|c|c|}
\hline $\begin{array}{c}\text { Excitation \& } \\
\text { Configuration }\end{array}$ & $\begin{array}{c}f_{s} \\
{[\mathrm{MHz}]}\end{array}$ & $Q_{s}$ & $k_{t}^{2}$ & $\left.\begin{array}{c}W \\
{[\mu \mathrm{m}]}\end{array}\right]$ & $n$ & $\begin{array}{c}L \\
{[\mu \mathrm{m}]}\end{array}$ & $\begin{array}{c}T \\
{[\mu \mathrm{m}]}\end{array}$ & $\begin{array}{c}\boldsymbol{K}_{\boldsymbol{i}} \\
{\left[\mathrm{ppm} / \mathrm{mA}^{2}\right]}\end{array}$ & $\left.\begin{array}{c}i_{c} \\
{[\mathrm{~mA}]}\end{array}\right]$ & \begin{tabular}{|c|}
$\begin{array}{c}i_{c, t} \\
{[\mathrm{~mA}]}\end{array}$ \\
\end{tabular} & $\left.\begin{array}{c}p_{c} \\
{[\mathrm{dBm}]}\end{array}\right]$ & $\begin{array}{c}p_{c} / A \\
{\left[\mu \mathrm{W} / \mu m^{2}\right]}\end{array}$ & $\begin{array}{c}p_{c} / V \\
{\left[\mu \mathrm{W} / \mu m^{3}\right]}\end{array}$ \\
\hline One-Port TFE & 177 & 2500 & $2.0 \%$ & 25 & 3 & 200 & 2 & -8.6 & 8.1 & 6.0 & 3.9 & 0.16 & 0.082 \\
\hline One-Port TFE & 222 & 1650 & $2.1 \%$ & 20 & 3 & 200 & 2 & -7.4 & 9.2 & 7.9 & 7.0 & 4 & .207 \\
\hline Two-Port TFE & 271 & 1150 & $1.6 \%$ & 15 & 4 & 200 & 2 & -23.7 & 5.2 & 5.3 & 9.4 & 0.73 & 0.364 \\
\hline Two-Port TFE & 3047 & 500 & $1.5 \%$ & 1.2 & 37 & 17 & 0.25 & -498.4 & 1.7 & 1.8 & -1.8 & 0.86 & 3.458 \\
\hline One-Port LFE & 1170 & 2400 & $1.0 \%$ & 4 & 15 & 80 & 3 & -48.5 & 2.4 & 2.6 & -1.0 & 0.16 & 0.055 \\
\hline One-Port LFE & 1643 & 1400 & $0.4 \%$ & 2 & 19 & 60 & 3 & -81.7 & 2.4 & 2.6 & 2.6 & 0.81 & 0.268 \\
\hline
\end{tabular}

$f_{s}$ : series resonant frequency of the resonator; $Q_{s}$ : quality factor at series resonant frequency; $k_{t}^{2}$ : effective electromechanical coupling coefficient $\kappa_{i}$ : current-frequency coefficient; $i_{c}:$ measured critical current; $i_{c, t}:$ theoretically calculated critical current; $p_{c}:$ measured critical power; $p_{c} / A$ : measured critical power per unit area; $p_{c} / V$ : measured critical power per unit volume

\section{EXPERIMENTAL RESULTS}

To validate the analytical equations presented in Section II and III, piezoelectric AlN CMRs were characterized in terms of current and power handling at different frequencies and for all the three electrode configurations. The resonators were probed directly in a probe station and their responses were measured by a network analyzer at different available power levels. Resonant frequency vs. actual driving current curves were plotted so as to extract the current-frequency coefficient $\left(\kappa_{i}\right)$ from Eq. (1).

With $Q$ and $\kappa_{i}$ known (both are experimentally measured), the theoretical critical current $\left(i_{c, t}\right)$ can be directly calculated using Eq. (10). The obtained $i_{c, t}$ value is used to verify that the one-dimensional isotropic model is sufficient to describe the power handling and nonlinear behavior of AlN CMRs. The main reason for not being able to predict the value of the nonlinear coefficients, $Y_{1}$ and $Y_{2}$, is that the AlN films are not well characterized. Especially, it is hard to predict these values when the AlN films are deposited by a sputtering method (instead of being a single-crystal grown material) and their properties are greatly affected by the deposition process.

The experimental results are listed in Table I. It is evident that the calculated $i_{c, t}$ values match well with those measured experimentally $\left(i_{c}\right)$, which verifies that the one-dimensional and isotropic model derived in this paper is sufficient to describe the general power handling behavior of AlN CMRs at different frequencies and with different electrode configurations. In other words, the model can be used to predict the general trend of the critical current (power) and define how it depends on the resonator physical parameters. However, due to the lack of apriori knowledge of $Y_{1}$ and $Y_{2}$ for different film thicknesses and deposition conditions, the equations cannot be used to calculate the exact values of critical current (power).

Nevertheless, using the experimentally measured $\kappa_{i}$ values, the nonlinear coefficient, $9 Y_{2}-10 Y_{1}^{2}$, can be extracted by using Eq. (9), and it is a direct indicator of the material nonlinearity. The data show that this coefficient is significantly degraded for the $250 \mathrm{~nm}$ thin-film two-port TFE AlN resonator (row 4 in Table I). This is not surprising since the rocking curve (FWHM) is $2.1^{\circ}$ for the thin AlN film, while it is less than $1.3^{\circ}$ for the other thicker films ( $>2 \mu \mathrm{m}$ ). If we compare the critical power density per area or volume within each of the three electrode configurations but at different frequencies, we find that the critical power density tends to increase with frequency. According to Eq. (12) and (13), this increase is due to the reduced $Q$ at higher frequencies and an increase in $f_{s}$. However, a simple comparison between the two-pot TFE $271 \mathrm{MHz}$ and $3047 \mathrm{MHz}$ resonators reveals that 10 times larger critical power density per unit volume can be obtained even in the presence of degraded material properties for thin film AIN and a decrease in the $Q$ by a factor of 2 . This clearly shows the trend of higher power handling density with higher frequency of operation.

\section{CONCLUSOIN}

The mechanically-limited power (current) handling and nonlinearity characteristics of piezoelectric AIN CMRs have been studied analytically and experimentally for different frequencies of operation and electrode configurations. It is proven that the derived model describes the general trend of the nonlinear behavior of the CMRs. It is important to note that a net increase of power handling density per area or volume can be attained by device scaling (i.e. miniaturization) to higher frequencies of operation.

\section{REFERENCES}

[1] M. D. Howard, et al., 39th ASFC, pp. 491-503, 1985.

[2] F. Kubat, et al., Microelectronic Engineering, vol. 82, Dec 2005.

[3] J. D. Larson III, et al., 2000 IUS, pp. 869-874, Oct 2000.

[4] J. Wang, et al., MEMS 2004, pp. 641-644, Jan 2004.

[5] D. Weinstein, et al., IEDM 2007, pp.415-418, Dec 2007.

[6] P. J. Stephanou, et al., 2006 IUS, pp. 2401-2404, Oct 2006.

[7] M. Rinaldi, et al., MEMS 2009, pp. 916-919, Jan 2009.

[8] V. Kaajakari, et al., JMEMS, vol. 13, no. 5, Oct 2004.

[9] M. Agarwal, et al., IEDM 2005, pp. 286-289, Dec 2005.

[10] L. C. Shao, et al., JMM, vol. 18, no. 6, Jun 2008.

[11] R. Mahameed, et al., JMM, vol. 18, no. 10, Oct 2008.

[12] W.-T. Hsu, 40th Annual PTTI Meeting, pp. 135-146.

[13] R. Abdolvand, et al., 2007 IUS, pp. 608-611, Oct 2007.

[14] G. Piazza, et al., JMEMS, vol. 15, no.6, Dec 2006.

[15] L. D. Landau, et al., Mechanics, 3rd ed. Oxford, 1999.

[16] R. L. Filler, 39th ASFC, pp. 311-316, May 1985.

[17] C. Zuo, et al., 2008 IEEE IFCS, Hawaii, USA, May 2008.

[18] C. Zuo, et al., 2007 IEEE IUS, New York, USA, Oct 2007.

[19] C. Zuo, et al., EFTF-IFCS 2009, Besançon, France, Apr 2009.

[20] J. H. Kuypers, et al., 2008 IEEE IFCS, pp. 240-249, May 2008. 